Ann. Zootech., I972, 21 (2), I63-I73.

\title{
INFLUENCE DE LA MACHINE DE RÉCOLTE SUR LA VALEUR ALIMENTAIRE DES ENSILAGES
}

\author{
I. - RÉSUltats PRÉLIMINAIRES
}

\author{
J.-P. DULPHY et C. DEMARQUILLY
}

avec la collaboration technique de J. ANDrieu, J. M. Borssau

H. Bousguet, Jacqueline JAmot, Marie JAiller, et L. L'Hôtelıier

Station de Recherches sur l'Élevage des Ruminants, Contre de Recherches de Clermont-Ferrand, I. N.R.A., 63 - Saint-Genès-Champanelle

\section{RÉSSUMÉ}

Nous avons comparé les caractéristiques fermentaires, la digestibilité et l'ingestibilité de I 6 ensilages fabriqués en I970 à partir des mêmes plantes sur pied mais récoltées, soit avec une machine à fléaux (brins de Io à $25 \mathrm{~cm}$ de long), soit avec une machine à tambour hacheur (brins de 0,5 à $I, 5 \mathrm{~cm}$ de long). Sept comparaisons ont été effectuées en petits silos de $4 \mathrm{~m}^{3}$, une en grands silos de Ioo $\mathrm{m}^{3}$ (tabl. $\mathrm{I}$ ).

La digestibilité et l'ingestibilité de tous les ensilages ont été mesurées sur des moutons maintenus en cage à métabolisme durant l'hiver 1970-I97I. Les 2 ensilages réalisés en grands silos ont aussi été distribués à volonté comme seul fourrage (mais avec $2 \mathrm{~kg}$ d'aliment concentré) durant 5 mois à 2 lots de 9 génisses (tabl. 4).

Les ensilages hachés finement (récoltés avec la machine à tambour hacheur) ont en moyenne un $\mathrm{pH}$ plus faible $(4,0$ contre 4,6$)$, une teneur en acide lactique plus élevée $(6,4$ contre $3,8 \mathrm{p}$. Ioo) et une teneur en acide butyrique plus faible $(0,3$ contre $3,6 \mathrm{p}$. Ioo) que ceux hachés grossièrement (récoltés avec la machine à fléaux). L'ammoniogenèse y est aussi beaucoup moins importante ( I 2,3 contre 2I, I p. Ioo d'azote sous forme ammoniacale) (tabI. 2).

Les ensilages hachés finement n'ont pas toujours été plus digestibles que ceux hachés grossièrement mais ils ont tous été ingérés en beaucoup plus grande quantité : en moyenne $50,5 \mathrm{~g}$ de matière sèche par $\mathrm{kg} \mathrm{P}^{\mathbf{0}^{\prime 75}}$ contre $3 \mathrm{I}, 4 \mathrm{~g}$ soit une augmentation de $6 \mathrm{I} \mathrm{p}$. 100 (22 à I $39 \mathrm{p}$. Ioo suivant les fourrages) (tabl. 3). Le hachage à la sortie du silo des ensilages récoltés à la machine à fléaux a permis d'augmenter de façon importante la quantité ingérée de ces ensilages mais celle-ci est encore très inférieure (de 35 p. roo) à celle ingérée quand le fourrage avait été haché avant la mise en silo.

Les génisses recevant l'ensilage haché finement ont ingéré en moyenne un peu plus de matière sèche que celles recevant l'ensilage lacéré : I,72 contre $I, 59 \mathrm{~kg}$ par Ioo $\mathrm{kg}$ de poids vif soit une différence de $8 \mathrm{p}$. Ioo seulement mais il est vrai que l'ensilage haché finement réalisé en grand silo était le seul à avoir des caractéristiques fermentaires plus mauvaises que son homologue récolté avec la machine à fléaux car une panne de la récolteuse à tambour hacheur avait interrompu pendant 2 jours le remplissage du silo. Il a cependant permis aux génisses de réaliser des croissances journalières significativement $(\mathrm{P}<\mathrm{o,OI})$ plus élevées qu'avec l'ensilage lacéré : 7 Io $\mathrm{g}$ contre $589 \mathrm{~g} /$ jour (tabl. 4). 
La machine de récolte est donc un élément très important à prendre en considération pour la réussite de l'ensilage. Un hachage fin du fourrage avant la mise en silos permet d'augmenter considérablement l'ingestibilité des ensilages, non seulement en favorisant une bonne fermentation au cours de la conservation, mais aussi en améliorant la "préhensibilité " des ensilages. Les résultats sont discutés.

\section{INTRODUCTION}

Un certain nombre d'études ont montré que le conditionnement (hachage ou lacération) des fourrages verts avant la mise en silos améliorait les caractéristiques fermentaires des ensilages et réduisait les pertes de matière sèche au cours de la conservation (DE MAN, I952, MARTIN et Bursse, I953 ; BALCH et al., I955; MuRdoch et al., I955; GoRdon et al., I958 ; Buysse, I96I ; MURDoch, I965). Il en résulte une amélioration de la digestibilité des ensilages (BALch et al., I955; MURDOch, I965), de la quantité ingérée (GORDON et al., I958; MURDOCH, r965) et des performances animales (Morrison, I960). Les améliorations constatées sont en général plus importantes avec le hachage qu'avec la lacération. Le but de ces études était essentiellement de comparer les ensilages fabriqués à partir de fourrages longs ou plus ou moins hachés et lacérés ; la finesse de hachage et le type de machine employée pour le conditionnement ne sont en général pas mentionnés.

Il existe actuellement trois grands types de machine de récolte et de conditionnement des fourrages destinés à l'ensilage :

- les récolteuses à fléaux : les fléaux fauchent le fourrage en même temps qu'ils le lacèrent en brins de Io à $30 \mathrm{~cm}$,

- les récolteuses à couteaux sur plateau : ces machines équipées d'une barre de coupe ou d'un pick-up, hachent le fourrage à l'aide de couteaux portés par un volant. La longueur moyenne des brins n'est jamais inférieure à $5 \mathrm{~cm}$;

- les récolteuses à couteaux sur tambour : ces machines équipées d'une barre de coupe ou d'un " pick-up ", hachent très finement et très régulièrement le fourrage à l'aide de couteaux portés par un tambour tournant à vitesse élevée. La longueur des brins peut être de $0,5^{-I} \mathrm{~cm}$. Ces machines qui étaient utilisées classiquement pour la récolte des fourrages destinés à la déshydratation à haute température, le sont de plus en plus aussi pour la récolte de l'ensilage de maîs ;

Ces trois types de machines ont été utilisés de I966 à I969 pour fabriquer les 87 ensilages expérimentaux de graminées et de trèfles violets dont nous avons étudié la composition chimique et mesuré la valeur alimentaire sur des moutons. Il ressort des résultats obtenus (DEMARQUILIY, en préparation) que la diminution des quantités ingérées entre la plante sur pied et la plante ensilée est beaucoup plus faible (environ 25 contre $40 \mathrm{p}$. IOO) quand la plante est récoltée avec une récolteuse à tambour hacheur (c'est-à-dire hachée en brins très courts 0,5 à $I, 5 \mathrm{~cm}$ ) qu'avec les autres machines. Ce résultat est cependant critiquable car ce n'étaient pas les mêmes fourrages qui avaient été récoltés avec les différentes machines de récolte. Aussi, avons-nous comparé en r970 les caractéristiques fermentaires, la digestibilité et 1'ingestibilité des ensilages fabriqués à partir des mêmes plantes sur pied et récoltées, soit avec une machine à fléaux, soit avec une machine à tambour hacheur. Sept comparaisons 
ont été effectuées en petits silos expérimentaux de $4 \mathrm{~m}^{3}$ au C. R. Z. V. de Theix et au domaine du Pin-au-Haras (Orne) ; une comparaison a été effectuée en grands silos de roo $\mathrm{m}^{3}$ au domaine d'Orcival (Puy-de-Dôme).

\section{MATÉRIEI, ET MÉTHODES}

\section{Ensilages réalisés}

A partir des 4 fourrages sur pied : une fétuque élevée $L P$ et une fétuque élevée Ludion à Theix, une herbe de prairie naturelle au Pin-au-Haras et une fléole à Orcival, nous avons fabriqué I 6 ensilages, I 4 en petits silos-tours de $4 \mathrm{~m}^{3}$ en bois gainé de plastique ou en butyl, 2 en siloscouloirs bétonnés de $100 \mathrm{~m}^{3}$ (tabl. I). La digestibilité et l'ingestibilité chez le Mouton des fourrages sur pied n'ont pu être mesurées que pour la fétuque $L P$ et l'herbe de prairie naturelle. Le même fourrage sur pied a été récolté :

- soit avec une ensileuse à tambour hacheur (type New-Holland) réglée pour hacher en brins de 0,5 à $\mathrm{I}, 5 \mathrm{~cm}$;

- soit avec une ensileuse à fléaux (type Kühn à Theix et à Orcival, type Ernterotor au Pin) quand il était ensilé directement et avec une ensileuse à plateau hacheur (type Koela) quand il était ensilé après préfanage. Le fourrage récolté avec les ensileuses à fléaux se présentait en brins de ro à $25 \mathrm{~cm}$ de long, celui récolté avec l'ensileuse à plateau hacheur en brins de 5 à I $5 \mathrm{~cm}$.

Le fourrage a été ensilé, soit immédiatement après la coupe sans ou avec addition d'acide formique ( $\mathrm{i}, 5$ litre d'acide à $80 \mathrm{p}$. Ioo par tonne), soit après un préfanage jusqu'environ 30 p. Ioo de matière sèche. Il a été tassé dans le silo au fur et à mesure du remplissage et, dès la fin de celui-ci, a été couvert d'un film de plastique et chargé de pierres (petits silos bois) ou de paille (silos-couloirs). Pour les silos butyl, l'enveloppe était simplement refermée.

\section{Mesures}

Nous avons mesuré sur 6 béliers adultes de race Texel, la digestibilité et l'ingestibilité de ces ensilages durant l'hiver qui a suivi leur récolte. Les animaux recevaient 2 repas par jour à volonté, à $8 \mathrm{~h}$ et à $\mathrm{I} 7 \mathrm{~h}$, les refus (environ Io $\mathrm{p}$. Ioo) étant enlevés avant la distribution du repas de 8 heures. Chaque période de mesure était de 6 jours et était précédée d'une période préexpérimentale de I $5_{5}$ jours.

Les 2 ensilages de fléole réalisés en grands silos ont aussi été distribués à volonté (Io p. Ioo de refus) comme seul fourrage à 2 lots de 9 génisses appariées 2 à 2, du I2 novembre I970 au 8 avril 197I. Le dispositif expérimental employé est rapporté dans le tableau 4. Les animaux de race Frisonne et Montbéliarde étaient âgés au départ de 305 jours et pesaient $242 \mathrm{~kg}$ en moyenne; ils ont été maintenus en stabulation libre sur paille et ont reçu, outre les 2 repas d'ensilage (9 et 15 heures), $2 \mathrm{~kg}$ d'aliment concentré par animal. Les quantités d'ensilage ingérées ont été mesurées pendant 3 jours chaque semaine. Les animaux ont été pesés deux jours de suite, ro jours après le début puis au milieu et à la fin de chaque période.

\section{Analyses}

Les échantillons secs représentatifs des fourrages offerts, des fourrages refusés et des fèces correspondant à chaque période de mesure de la digestibilité ont été analysés pour déterminer leur teneur en cendres, en matières azotées $(\mathrm{N} \times 6,25)$ et en cellulose brute Weende.

Un échantillon représentatif de l'ensilage distribué aux moutons a été prélevé au début et à la fin de chaque période de mesure de la digestibilité. Sur ces échantillons frais, nous avons déterminé la teneur en azote et sur leur jus nous avons mesuré le $\mathrm{pH}$ et déterminé les teneurs en ammoniaque (CoNway, I957), en acide lactique (BARKER et Summerson, I94I) et en acides gras volatils par chromatographie en phase gazeuse (RIGAUD et JouRneT, I970). La teneur en acides gras volatils des ensilages du Pin a cependant été déterminée par la méthode de Lepper et sur un seul échantillon prélevé au milieu de chaque période de mesure. 


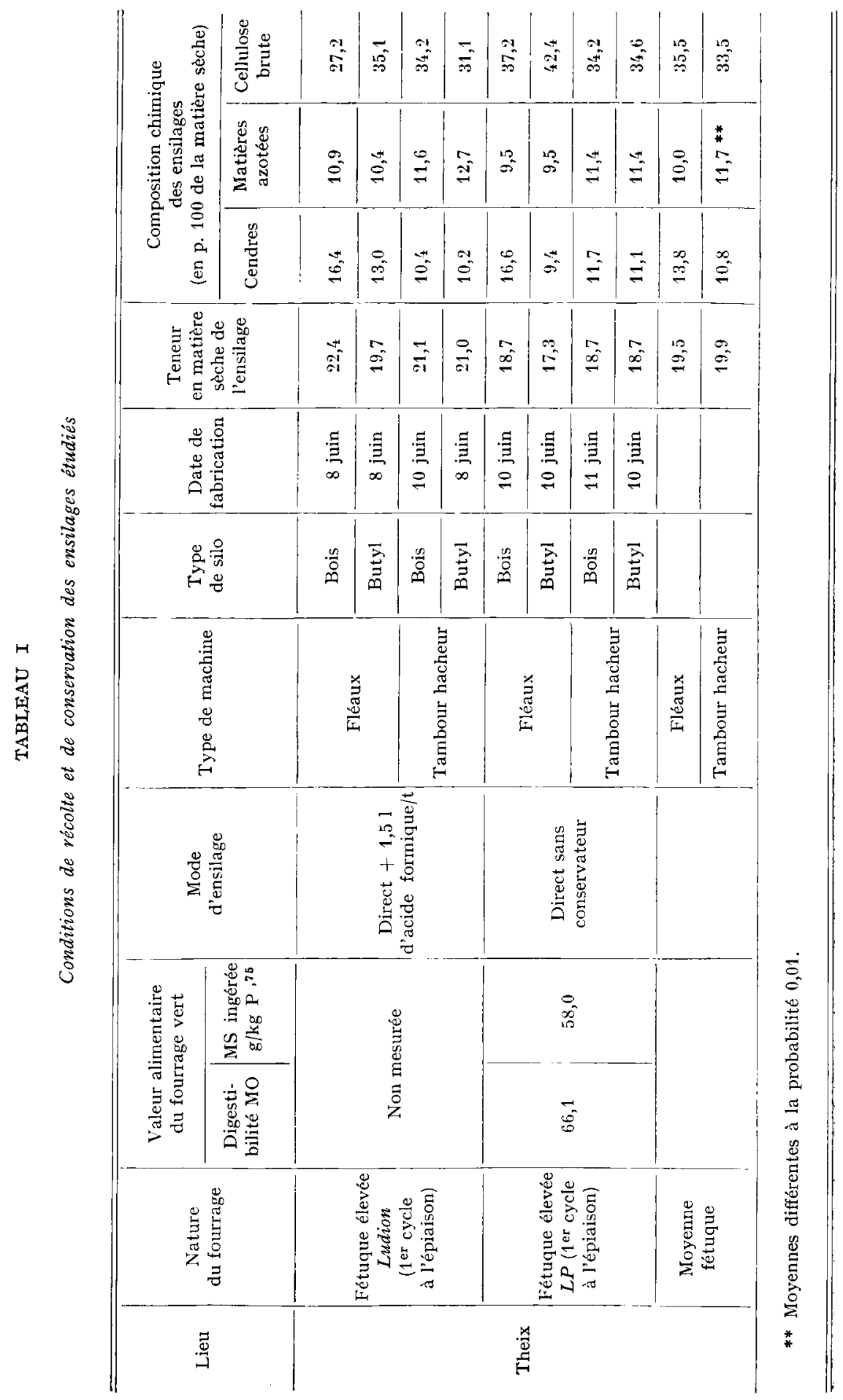




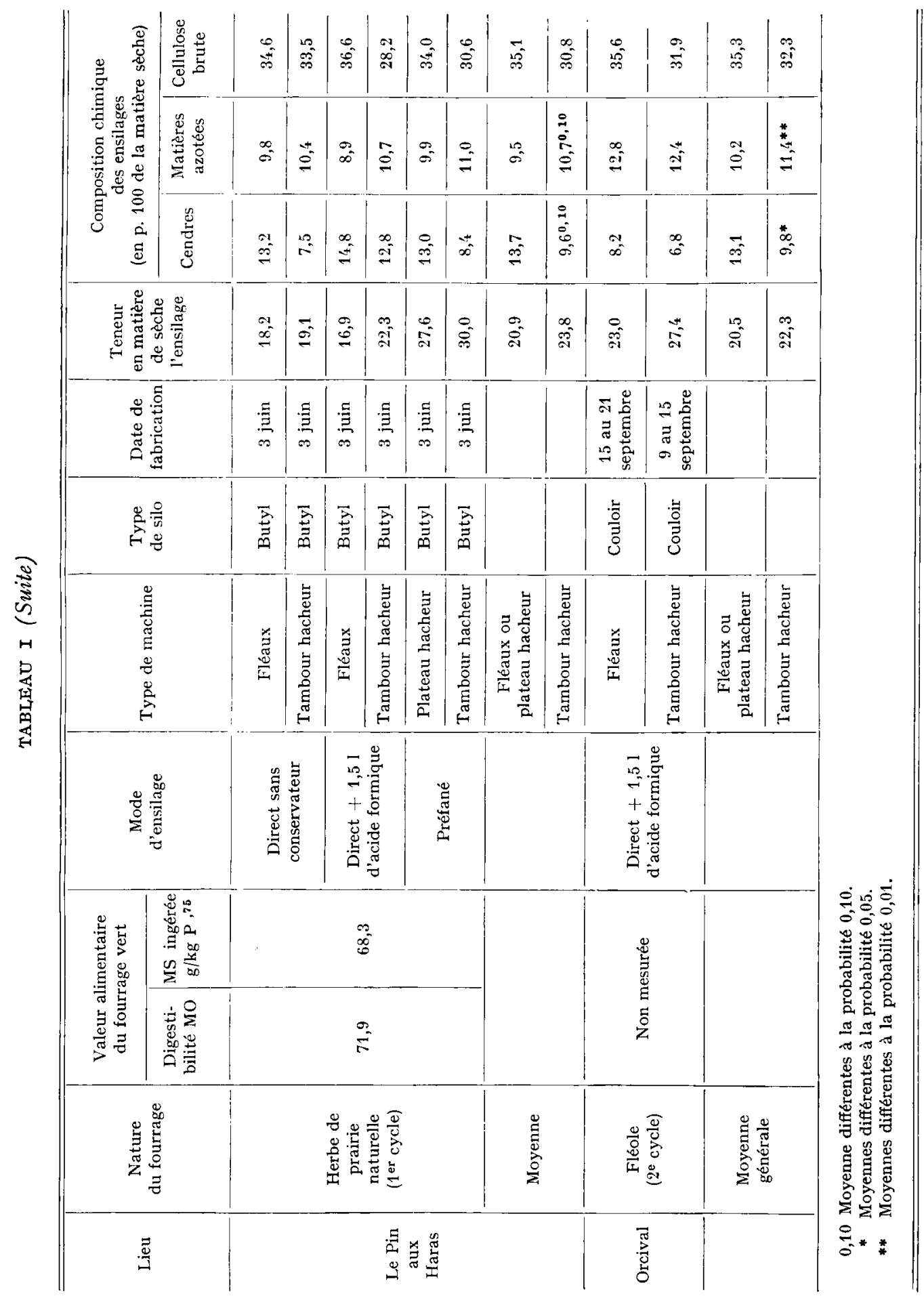




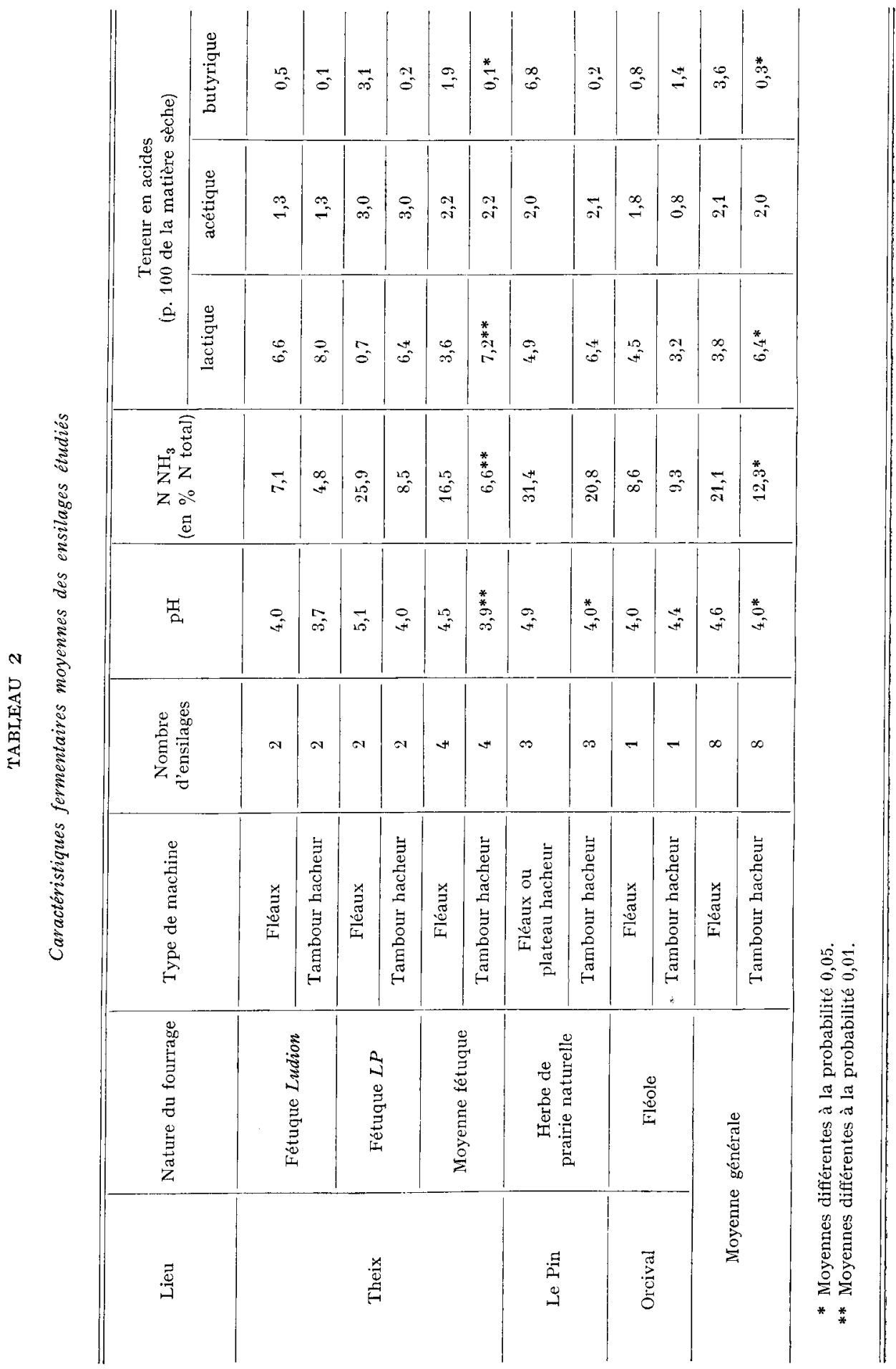




\section{RÉSULTATS}

\section{Composition chimique et caractéristiques fermentaires}

La teneur en cendres des ensilages récoltés à la machine à fléaux est systématiquement plus élevée que celle des ensilages récoltés avec la machine à tambour hacheur, (tabl. I) ce qui indique une contamination beaucoup plus importante du fourrage avec la terre lors de la récolte. De même, les ensilages récoltés à la machine à fléaux sont légèrement plus pauvres en matières azotées et plus riches en cellulose brute, ce qui doit refléter des pertes plus importantes lors de la conservation.

Les caractéristiques fermentaires de ces ensilages sont d'ailleurs bien plus mauvaises (tab1. 2). Ils ont en moyenne un pH plus élevé $(4,6$ contre 4,0$)$, une teneur en acide lactique plus faible $(3,8$ contre $6,4 \mathrm{p}$. IOo) et une teneur nettement plus élevée en acide butyrique (3,6 contre $0,3 \mathrm{p}$. IOo). Enfin, l'ammoniogenèse $\mathrm{y}$ est beaucoup plus importante (2I,I p. Ioo d'azote sous forme ammoniacale contre I2,3 p. IOo). Ces différences se retrouvent systématiquement quel que soit le fourrage considéré (sauf la fléole vraisemblablement parce qu'une panne de la récolteuse à tambour hacheur a interrompu pendant 2 jours le remplissage du silo), mais elles sont plus grandes en l'absence qu'en présence de conservateur (acide formique), notamment pour les fétuques. Avec ces dernières, il y a en effet, une interaction hautement significative $(\mathrm{P}<\mathrm{O}, \mathrm{OI})$ entre la machine de récolte et le conservateur, l'addition d'acide formique ayant d'ailleurs entraîné, elle aussi, une amélioration hautement significative $(\mathrm{P}<0,0 \mathrm{I})$ de la qualité de la conservation. En revanche, il n'y a pas d'interaction entre la machine de récolte et le type de silo, la qualité de la conservation n'ayant été que faiblement améliorée $(\mathrm{P}<\mathrm{O}, \mathrm{IO})$ par l'emploi de silo butyl par comparaison au silo en bois gainé de plastique.

\section{Digestibilité et ingestibilité mesurées sur les moutons}

Les ensilages de fétuque élevée ont eu la même digestibilité quelle que soit la machine de récolte mais ceux hachés finement avec la récolteuse New Holland ont été ingérés en quantité bien plus élevée, en moyenne $62,8 \mathrm{~g}$ contre $26,3 \mathrm{~g} / \mathrm{kg} \mathrm{P}^{0,75}$ pour la fétuque Ludion, 44,3 g contre $26,3 \mathrm{~g}$ pour la fétuque LP (tab1. 3). Ceux ensilés en silos butyl ont été légèrement plus digestibles que ceux ensilés en silos en bois $(68, \mathrm{I}$ contre $66,3 \mathrm{P}<0, \mathrm{I} 0)$ mais n'ont pas été ingérés en plus grande quantité $(40,9$ contre $38,4 \mathrm{~g})$. En revanche, les ensilages d'herbe de prairie permanente hachés finement ont été à la fois plus digestibles (digestibilité de la matière organique de 64,9 contre $60,8 \mathrm{p}$. Ioo) et ingérés en plus grande quantité (48,I contre 36 ,I g). Quant à l'ensilage de fléole haché finement il a été un peu moins digestible (vraisemblablement à cause de l'interruption du remplissage), mais il a cependant été ingéré en quantité un peu plus élevée : 45,6 g contre $37,4 \mathrm{~g}$.

Les différences d'ingestibilité observées sont toutes significatives $(\mathrm{P}<0,05$ ou o,or). En revanche, les différences de digestibilité, quand elles existent, ne le sont pas mais il est vrai que le test de signification n'a été effectué que sur une valeur par échantillon car nous n'avons pas déterminé la teneur en cendres des fèces de chaque mouton mais celles de l'échantillon représentatif des 6 moutons.! 
En moyenne pour la fétuque $L P$ et 1 'herbe de prairie permanente dont nous connaissions la digestibilité et l'ingestibilité en vert, l'ensilage a entraîné une diminution de digestibilité et d'ingestibilité respectivement de 4,7 points et $27 \mathrm{p}$. Ioo quand le fourrage mis dans le silo avait été haché finement et de 6,2 points et $50 \mathrm{p}$. I00 quand le fourrage avait été haché plus grossièrement.

TABLEAU 3

Digestibilité et ingestibilité moyennes des ensilages étudiés

\begin{tabular}{|c|c|c|c|c|c|c|}
\hline \multirow{2}{*}{ Lieu } & \multirow{2}{*}{$\begin{array}{l}\text { Nature du } \\
\text { fourrage }\end{array}$} & \multirow{2}{*}{ Type de machine } & \multirow{2}{*}{$\begin{array}{c}\text { Nombre } \\
\text { d'ensilages }\end{array}$} & \multirow{2}{*}{$\begin{array}{c}\text { Digestibilité de } \\
\text { la matière orga- } \\
\text { nique (p. 100) }\end{array}$} & \multicolumn{2}{|c|}{$\begin{array}{c}\text { Quantité de matière } \\
\text { sèche ingérée }\end{array}$} \\
\hline & & & & & $\begin{array}{l}\mathrm{P} 0,75 \\
\mathrm{~g} / \mathrm{kg}\end{array}$ & $\begin{array}{c}\text { (p. } 100 \\
\text { du fléau) }\end{array}$ \\
\hline \multirow{4}{*}{ Theix } & \multirow{2}{*}{$\begin{array}{l}\text { Fétuque } \\
\text { Ludion }\end{array}$} & Fléaux & 2 & 69,3 & 26,3 & 100 \\
\hline & & Tambour hacheur & 2 & 68,7 & $62,8^{* *}$ & 239 \\
\hline & \multirow{2}{*}{$\begin{array}{l}\text { Fétuque } \\
\qquad L P\end{array}$} & Fléaux & 2 & 65,5 & 26,3 & 100 \\
\hline & & Tambour hacheur & 2 & 65,2 & $4\{, 3 * *$ & 168 \\
\hline \multirow[t]{2}{*}{ Le Pin } & \multirow{2}{*}{$\begin{array}{l}\text { Herbe de } \\
\text { prairie } \\
\text { naturelle }\end{array}$} & $\begin{array}{c}\text { Fléaux ou plateau } \\
\text { hacheur }\end{array}$ & 3 & 60,9 & 36,1 & 100 \\
\hline & & Tambour hacheur & 3 & 64,2 & $48,1 * *$ & 133 \\
\hline \multirow{2}{*}{ Orcival } & \multirow{2}{*}{ Fléole } & Fléaux & 1 & 64,9 & 37,4 & 100 \\
\hline & & Tambour hacheur & 1 & 61,4 & $45,6^{*}$ & 122 \\
\hline \multirow{2}{*}{\multicolumn{2}{|c|}{ Moyenne générale }} & $\begin{array}{c}\text { Fléaux ou plateau } \\
\text { hacheur }\end{array}$ & 8 & 64,6 & 31,4 & 100 \\
\hline & & Tambour hacheur & 8 & 65,2 & $50,5 * *$ & 161 \\
\hline
\end{tabular}

* : Moyennes différentes à la probabilité 0,05 .

** : Moyennes différentes à la probabilité 0,01 .

Les 2 ensilages de fétuque Ludion récoltés à la machine à fléaux ont aussi été distribués aux moutons après avoir été hachés finement dans un hache-paille à la sortie du silo. Le hachage a posteriori a permis d'augmenter de façon importante la quantité ingérée (en moyenne de 26,3 à 40,6 g soit une augmentation de 54 p. IOo) mais celle-ci est encore très inférieure (de $22,2 \mathrm{~g}$ soit $35 \mathrm{p}$. Ioo) à celle ingérée quand le fourrage avait été haché avant la mise en silo. En revanche, la digestibilité a diminué de 2,7 points vraisemblablement à cause de l'augmentation du niveau d'ingestion.

Quantités ingérées par les génisses et gain de poids vif

L,es génisses recevant l'ensilage de fléole haché finement ont ingéré en moyenne un peu plus de matière sèche que celles recevant l'ensilage de fléole lacéré : 1,72 contre I,59 $\mathrm{kg}$ par roo $\mathrm{kg}$ de poids vif soit une différence de 8 p. Ioo (tab1. 4). N'ayant pas 
enregistré les consommations individuelles il n'est pas possible de savoir si cette différence est significative ou non. Elle est cependant inférieure à celle enregistrée sur les moutons (22 p. IOo) mais cette dernière porte sur une période beaucoup plus courte : 6 jours contre $\mathrm{I} 34$ jours pour les génisses. Il est cependant possible que les génisses soient moins sensibles que les moutons à la "qualité " et à la finesse de hachage de l'ensilage qui leur est distribué. L'aliment concentré qui a représenté en moyenne 26 p. Ioo de la quantité totale de matière sèche ingérée par les génisses a dû, lui aussi, contribuer à tamponner les différences de quantités ingérées entre les 2 ensilages. L'ensilage finement haché a cependant permis aux génisses de réaliser des croissances journalières significativement $(\mathrm{P}<\mathrm{O}, \mathrm{or})$ plus élevées que l'ensilage lacéré : 7 Io g contre $589 \mathrm{~g} /$ jour, soit une différence de I2I g par jour (20 p. IOo).

\section{TABLEAU 4}

Influence de la machine de récolte sur la quantité d'ensilage de fléole ingérée et sur le gain de poids vif journalier des génisses

\begin{tabular}{|c|c|c|c|c|c|}
\hline & \multicolumn{2}{|c|}{ Lot 1} & \multicolumn{2}{|c|}{ Lot 2} & Moyenne \\
\hline \multicolumn{6}{|l|}{ Caratéristiques des animaux } \\
\hline Nombre $\ldots \ldots \ldots \ldots \ldots \ldots \ldots \ldots \ldots$ & \multicolumn{2}{|c|}{9} & \multicolumn{2}{|c|}{9} & \\
\hline Age à la mise en lot $(12-11-70)$ (jours) $\ldots \ldots \ldots$ & \multicolumn{2}{|c|}{305} & \multicolumn{2}{|c|}{305} & \\
\hline Poids initial $(12-11-70)(\mathrm{kg}) \ldots \ldots \ldots \ldots \ldots$ & \multicolumn{2}{|c|}{242} & \multicolumn{2}{|c|}{242} & \\
\hline \multicolumn{6}{|l|}{ Dispositif expérimental et durée des périodes } \\
\hline Période $1\left(P_{1}\right)$ 22-11-70 au 31-01-71 $\ldots \ldots \ldots$ & \multirow{2}{*}{\multicolumn{2}{|c|}{$\begin{array}{l}\text { Ensilage haché } \\
\text { Ensilage lacéré }\end{array}$}} & \multirow{2}{*}{\multicolumn{2}{|c|}{$\begin{array}{l}\text { Ensilage lacéré } \\
\text { Ensilage haché }\end{array}$}} & \\
\hline Période $2\left(P_{2}\right)$ 01-02-71 au 08-04-71 $\ldots \ldots \ldots$ & & & & & \\
\hline \multicolumn{6}{|l|}{$\begin{array}{l}\text { Quantités de matic̀re sèche ingérées } \\
(\mathrm{kg} \text { pour } 100 \mathrm{~kg} \text { poids vif) }\end{array}$} \\
\hline \multirow{2}{*}{ Ensilage $\quad\left\{\begin{array}{l}\text { finement haché } \ldots \\
\text { lacéré } \ldots \ldots \ldots \ldots\end{array}\right.$} & \multirow{2}{*}{$\begin{array}{l}\mathrm{P}_{1} \\
\mathrm{P}_{2}\end{array}$} & \multirow{2}{*}{$\begin{array}{l}1,81 \\
1,67\end{array}$} & \multirow{2}{*}{$\begin{array}{l}\mathrm{P}_{2} \\
\mathrm{P}_{1}\end{array}$} & \multirow{2}{*}{$\begin{array}{l}1,64 \\
1,52\end{array}$} & \multirow{2}{*}{$\begin{array}{l}1,79 \\
1,59\end{array}$} \\
\hline & & & & & \\
\hline Ensilage + f finement haché .. & $P_{1}$ & $2,4^{\prime}+4$ & $\mathrm{P}_{2}$ & 2,18 & 2,31 \\
\hline aliment concentré $\{$ lacéré...$\ldots \ldots \ldots$ & $\mathrm{P}_{2}$ & 2,20 & $\mathrm{P}_{1}$ & 2,15 & 2,17 \\
\hline Gain de poids vif (g/jour/génisses) & & & & & \\
\hline Ensilage finement haché (New Holland) .... & $P_{1}$ & 713 & $\mathrm{P}_{2}$ & 708 & 710 \\
\hline Ensilage lacéré (fléaux) $\ldots \ldots \ldots \ldots \ldots \ldots$ & $P_{2}$ & 568 & $P_{1}$ & 610 & 589 \\
\hline
\end{tabular}

\section{DISCUSSION}

La machine de récolte est donc un élément très important à prendre en considération pour la réussite d'un ensilage.

Les récolteuses à fléaux contaminent souvent les fourrages avec de la terre, ce qui favorise l'ensemencement en bactéries butyriques et n'assurent pas un hachage suffisamment fin. Les fourrages hachés en brins très courts peuvent plus facilement, d'une part se tasser, ce qui assure une anaérobiose plus ra pide et meilleure, d'autre 
part libérer leurs sucs cellulaires. La fermentation lactique démarre donc rapidement et amène le $\mathrm{pH}$ en-dessous de 4,0, inhibant ainsi la fermentation butyrique et limitant l'ammoniogenèse. Ces résultats sont en accord avec ceux obtenus par de nombreux auteurs (voir introduction).

Il en résulte une réduction des pertes de matière sèche et de valeur nutritive au cours de la conservation, observations déjà faites par $\mathrm{BALCH}$ et al., I955 et par MuRDOCH, I965. La réduction des pertes de valeur nutritive est d'ailleurs certainement sousévaluée dans nos résultats car les mesures de digestibilité ont été effectuées sur des moutons alimentés à volonté. L a digestibilité des ensilages récoltés avec la machine à fléaux et qui ont été ingérés en faible quantité a donc été surestimée par rapport à celle des ensilages récoltés avec la machine à tambour hacheur et qui ont été consommés en quantité beaucoup plus élevée. C'est notamment le cas pour les ensilages de fétuque dont la digestibilité a d'ailleurs diminué quand un hachage a posteriori a permis d'augmenter leur ingestibilité.

L'influence de la machine de récolte a surtout été spectaculaire sur les quantités ingérées et cela que les ensilages soient réalisés avec ou sans conservateur et conservés dans des silos hermétiques ou non hermétiques. Ce résultat est très important car l'ingestibilité souvent faible des ensilages d'herbe constitue un des facteurs limitant essentiel de leur valeur alimentaire. Un hachage fin du fourrage avant la mise en silo permet d'augmenter considérablement 1'ingestibilité des ensilages, non seulement en favorisant une bonne fermentation au cours de la conservation, mais aussi en améliorant la préhensibilité des ensilages. L'influence de la préhensibilité sur 1'ingestibilité des ensilages avait déjà été mise en évidence par MuRDoch (I965) sur des vaches laitières et nous avons déjà essayé d'en expliquer le mode d'action (DutPHY et DeMarouilit, I97I).

Reçu pour publication en janvier 1972.

\author{
SUMMARY
}

EFFECT OF TYPE OF FORAGE HARVESTER ON THE FEEDING VALUE OF SMAGES. I. - PREIIMINARY RESUITS

The aim of the present study was to compare fermentation characteristics, digestibility and voluntary intake of 16 silages produced in 1970 from the same original herbage, but harvested with either a flail hervester (Io to $25 \mathrm{~cm}$ lenght material) or precision-chop forage harvester $\left(0.5\right.$ to $\mathrm{I} .5 \mathrm{~cm}$ lenght material). Seven comparisons were made with small silos $\left(4 \mathrm{~m}^{3}\right)$ and one with large silos ( $\left.100 \mathrm{~m}^{3}\right)$ (table. I).

Digestibility and voluntary intake of all silages were measured with sheep housed in metabolism crate during the winter of I970-1971. 2 silages conserved in large silos were also offered ad libitum as the only forage (with $2 \mathrm{~kg}$ of concentrate feed) to 2 groups of 9 heifers for 6 months (table. 4).

Finely chopped silages (harvested with a precision-chop forage harvester) showed lower mean $\mathrm{pH}$ values (4.o versus 4.6$)$, higher lactic acid contents $(6.4$ versus $3.8 \mathrm{p}$. Ioo) and lower butyric acid contents ( 0.3 versus $3.6 \mathrm{p}$. Ioo) than coarsely chopped silages (harvested with a flail harvester). The $\mathrm{NH}_{3}$ fraction $\left(\mathrm{NH}_{3}-\mathrm{N}\right.$ expressed as p. Ioo of total $\left.\mathrm{N}\right)$ was also much lower in finely chopped silages (12.3 versus 2 I. I p. Ioo nitrogen in the form of ammonia) (table 2 ).

Finely chopped silages were not always more digestible than the coarsely chopped ones, but their voluntary intake was much higher : on an average $50.5 \mathrm{~g}$ (dry matter per $\mathrm{kg} \mathrm{P}^{\mathbf{0 . 7 5}}$ ) and 
$3 \mathrm{I} .4 \mathrm{~g}$ respectively, i.e. an increase of $6 \mathrm{I}$ p. Ioo (22 to I39 p. Ioo according to the silage) (tabl. 3 ). If the silages harvested with the flail harvester were chopped after ensiling, their voluntary intake was greatly increased, but still remained much lower (35 P. Ioo) than the intake of forages chopped before ensiling.

The mean dry matter intake of the heifers receiving finely chopped silage was a little higher than that of the animals fed coarsely chopped silage : I.72 vorsus $1.59 \mathrm{~kg}$ per Ioo $\mathrm{kg}$ live weight, i.e. a difference of only $8 \mathrm{p}$. Ioo. However, it must be noted that the finely chopped silage stored in the large silo was the only one showing poorer fermentation characteristics than silos containing herbage harvested with the flail harvester, and the resason was a 2 day interruption during filling of the silo caused by a failure of the precision-chop forage harvester. Nevertheless, heifers fed this silage showed significantly higher daily growth rates $(\mathrm{P}<0.0 \mathrm{I})$ than those offered coarsely chopped silage : 7 I0 $\mathrm{g}$ and $589 \mathrm{~g} /$ day respectively (table. 4).

Consequently, the type of harvester plays a very important role in successful production of silages. Fine chopping of the forage before ensiling considerably increases the voluntary intake of silage, not only by favouring good fermentation during storage, but also by improving the "prehensibility " of the silages. The results are discussed.

\section{RÉFÉRENCES BIBLIOGRAPHIQUES}

Barker S. B., Summerson W. H., I94I. The colorimetric determination of lactic acid in biological material. J. Biol. Chem., 137, 535 .

Balch C. C., Murdoch J. C., Turner J., I955. The effect of chopping and lacerating before ensiling on the digestibility of silage by cows and steers. J. Brit. Grassl. Soc., 10, 326-329.

Buysse F., I96I. Le hachage influence-t-il l'ensilage? Revue de l'A griculture. $4^{\circ}$ année ${ }^{\circ} 6,773^{-} 778$.

Conway E. J., 1957. Microdiff usion analysis and volumetric error. London: Crosby, Lockwood. London.

De MAN J. C., I952. The influence of crushing on the pH of grass silage. Nature, Lond, 19, $246-247$.

Demarquilly C., I972. Valeur alimentaire des ensilages de graminées et légumineuses fourragères (en préparation).

Dulphy J.rP., Demarguilly C., I97r. Liaisons entre le comportement des moutons pendant l'ingestion et les quantités de fourrage ingérées. $X^{\mathrm{e}}$ Congrès Int. Zootech., Versailles, I 7-23 juillet.

Dulphy J.-P., r97r. Influence du poids vif et du niveau d'ingestion sur le comportement alimentaire et mérycique du mouton. Ann. Zootech, 20 477-486.

Gordon C. H., Melin C. G., Wiseman H. G., Irvin H. M., Mc Calmont J. R., i958. Chemical quality, nutrient preservation and feeding value of silages stored in bunker silos. J. Dairy Sci., 41, I738-I746.

Martin J., Buysse F., I953. Ensiling experiments with wilted lucerne and the influence of chopping on the preservation process. Meded-Landb. Hogesch., Gent., 17, 565-59r.

Morrison J., I960. Recent developments in the production and feeding of grass silage. Proc. 8th Int. Grassl. Congr., 693-697.

Murdoch J. C., Balch D. A., Holdsworth M. C., Wood M., 1955. The effect of chopping and lacerating and wilting herbage on the chemical composition of silage. J. Brit. Grassl. Soc., 10, I8 I-I 88.

Murdoch J. C., 1965. The effect of lenght of silage on its voluntary intake by cattle. J. Brit. Grassl. Soc., 70, 54-58.

Rigaud J., Journet M., ig7o. Méthode de dosage des acides gras volatils dans le liquide du rumen. Ann. Biol. anim. Bioch. Biophys., 10, I5x-I57. 